\title{
Safety and Immunogenicity of Live Oral Cholera Vaccine CVD 103-HgR in Children and Adolescents Aged 6-17 Years
}

\author{
James M. McCarty, ${ }^{1 \star}$ Emma C. Gierman, ${ }^{2}$ Lisa Bedell, ${ }^{2}$ Michael D. Lock, ${ }^{2}$ and Sean Bennett ${ }^{2}$ \\ ${ }^{1}$ Stanford University School of Medicine, Stanford, California; ${ }^{2}$ PaxVax, Inc., Redwood City, California
}

\begin{abstract}
The attenuated recombinant Vibrio cholerae O1 vaccine strain CVD 103-HgR, redeveloped as PXVX0200, elicits a rapid serum vibriocidal antibody (SVA) response and protects against cholera-induced diarrhea in adult volunteer challenge trials but has not been studied in children in developed countries. We performed a phase 4, placebo-controlled, double-blind, multicenter study to assess the safety, immunogenicity, and tolerability of a single, oral dose of PXVX0200 in children and adolescents aged 6-17 years in the United States and bridged immunogenicity to adults aged 18-45 years from a separate lot consistency study. Volunteers were randomized to receive a single dose of $1 \times 10^{9}$ colony forming units (CFU) of PXVX0200 or placebo. Immunogenicity endpoints included SVA levels on days 1, 11, and 29 in volunteers aged 6-17 years and also on days 91 and 181 in volunteers aged 12-17 years. Safety was assessed by comparing solicited signs and symptoms on days 1-8, unsolicited adverse events (AEs) through day 29, and serious AEs through day 181. A total of 374 participants were enrolled, comprising 321 vaccine and 53 placebo recipients. The SVA seroconversion rates 10 days after immunization were $98.6 \%$ and $2.1 \%$ in vaccine and placebo recipients, respectively, and the vaccine seroconversion rate was non-inferior to the $93.5 \%$ rate seen in adults aged $18-45$ years. Most reactogenicity was mild to moderate, and there were no vaccine-related serious AEs. The complete dose was consumed in $95.3 \%$ and $98.1 \%$ of vaccine and placebo recipients, respectively. PXVX0200 appears safe, immunogenic, and well tolerated in children and adolescents aged $6-17$ years.
\end{abstract}

\section{INTRODUCTION}

Cholera is an acute enteric infection caused by the ingestion of water or food containing the bacterium Vibrio cholerae. ${ }^{1}$ It principally occurs in countries with insufficient access to safe water and proper sanitation, with even more dramatic impact in areas where basic environmental infrastructures are disrupted or have been destroyed. ${ }^{2}$ Cholera is characterized in its most severe form (cholera gravis) by a sudden onset of acute electrolyte-rich watery diarrhea that can lead to severe dehydration and death, an outcome seen most commonly in children younger than 5 years. ${ }^{1}$ The very short incubation period, several hours to 5 days, enhances the potentially explosive pattern of outbreaks.

The current worldwide cholera pandemic has been ongoing since 1961 and is caused by V. cholerae O1 El Tor. Cholera occurs in an endemic form in many developing countries and also in explosive outbreaks, as seen in South America in 1991, Haiti in 2010, and Yemen in 2016. ${ }^{2-4}$ It is estimated that 1.3-4.0 million cholera cases, with $21,000-143,000$ deaths, occur each year worldwide. ${ }^{5}$ Cholera also represents a risk to travelers to countries with endemic or epidemic cholera. ${ }^{6-8}$ The persistence of cholera in many countries in Asia and Africa, the appearance of particularly severe clinical disease due to El Tor strains expressing classical biotype cholera toxin, and the increasing prevalence of antimicrobial resistance make the control of cholera a high public health priority. ${ }^{9}$

Serum vibriocidal antibodies (SVAs) produced by natural or experimental $V$. cholerae infection correlate with protection against cholera. ${ }^{10,11}$ Experimental (challenge) infection in adults resulted in protective immunity against rechallenge with both homologous and heterologous strains that lasted for at least 3 years. ${ }^{12}$ This led to studies of a number of candidate deletion mutants of classical and $\mathrm{El}$ Tor $V$. cholerae $\mathrm{O} 1$

*Address correspondence to James M. McCarty, Stanford University School of Medicine, 291 Campus Dr., Stanford, CA 94305. E-mail: jmccart2@stanford.edu designed to produce similar immunity and resulted in the live, attenuated $V$. cholerae strain CVD 103-HgR as a safe and effective oral vaccine for the prevention of cholera. ${ }^{13}$ Clinical trial experience with CVD 103-HgR included administration to more than 27,000 adults and children as young as 3 months of age. ${ }^{14-23}$ CVD 103-HgR was licensed in several countries ex-United States under the trade names Orochol, Orochol E, and Mutachol, and more than 500,000 commercial doses of CVD 103-HgR vaccine were sold with an indication in travelers aged 2 years or older. ${ }^{24}$ Production was discontinued in 2001 for commercial reasons. CVD 103-HgR was acquired by PaxVax in 2009 and was redeveloped under the research name PXVX0200.

The safety, immunogenicity, and efficacy of PXVX0200 in adults were established in four randomized, double-blind, placebo-controlled, multicenter clinical trials. A phase 1 trial demonstrated that PXVX0200 was well tolerated with a SVA seroconversion rate of $89 \% .{ }^{25} \mathrm{~A}$ phase 3 cholera challenge trial of PXVX0200 demonstrated $90 \%$ protective efficacy at 10 days and $80 \%$ at 3 months versus placebo following ingestion of $1 \times 10^{5}$ wild-type $V$. cholerae O1 El Tor Inaba strain N16961, and also established SVA seroconversion as a correlate of protection against cholera diarrhea. ${ }^{26,27} \mathrm{~A}$ large phase 3 lot consistency study in 3,146 adults aged 18-45 years demonstrated $93.5 \%$ vibriocidal seroconversion 10 days after vaccination with PXVX0200 and further documented safety, whereas another phase 3 study in 398 volunteers aged 46-64 years demonstrated the immunogenicity and safety of PXVX0200 in older adults. ${ }^{28,29}$ As a result of these trials, PXVX0200 was approved by the United States Food and Drug Administration (FDA) in 2016 under the trade name Vaxchora ${ }^{\circledR}$ (PaxVax, Inc., Redwood City, CA) for use in adults aged 18 through 64 years traveling to cholera-affected areas.

Previous studies in children in developing countries using a single dose of the CVD 103-HgR vaccine strain showed the vaccine was well tolerated and suggested that the immune response, as measured by SVA seroconversion rates and geometric mean titers (GMTs), was lower than the response in 
adults. $^{14-24}$ Immune responses to oral vaccines may be impacted in infants and children in cholera-endemic countries because of natural exposure to infections, age-related differences in immune function, breastfeeding, and nutritional status. ${ }^{30-32}$ Although Orochol was used in children in Europe, Canada, and Australia, there are no published studies of the serological response rates in children in developed countries. Because SVA seroconversion following PXVX0200 vaccination was a strong correlate of protection in the adult challenge study, the FDA accepted that this measure could be used to bridge immunogenicity and presume efficacy in a pediatric population in an industrialized country. ${ }^{13}$ Therefore, this phase 4 study was performed to assess the safety, immunogenicity, and tolerability of a single oral dose of PXVX0200 in children and adolescents in a developed country and to bridge immunogenicity in children and adolescents to adults aged $18-45$ years from the lot consistency study. ${ }^{28}$

\section{METHODS}

Study design. This was a multicenter phase 4 randomized, double-blind, placebo-controlled trial designed to assess PXVX0200 immunogenicity, safety, palatability, and acceptability in children and adolescents aged $2-17$ years. The study was performed at seven U.S. sites from November 2017 through May 2018 in children aged 6-17 years and is ongoing in children aged 2-5 years. Results for children aged 6-17 years are presented here. The study included a screening period of 30 days and a blinding period from day 1 to day 181 . Two age cohorts, 12-17 years (cohort 1 ) and 6-11 years (cohort 2) were enrolled concurrently. Study protocols were reviewed by the institutional review board (IRB) at each site, and informed consent was obtained before screening from each participant's parent or legal guardian. Participants in cohort 1 provided written assent, whereas participants in cohort 2 provided written assent as per each IRB's recommendation. The study included healthy children aged 6-17 years without a significant medical history or physical examination findings at screening, as previously described in the adult lot consistency study. ${ }^{28}$ Travel was not restricted during the study. In female participants of childbearing potential, a urine pregnancy test was performed at screening and before vaccine administration. Within each cohort, eligible study participants were randomized 6:1 to receive either a single dose of PXVX0200 or a physiologic saline placebo.

Solicited reactogenicity signs and symptoms included daily temperature, presence of tiredness, headache, lack of appetite, nausea and vomiting, diarrhea, and abdominal pain on study days 1-8 collected using a memory aid (diary card). On day 11 , blinded study staff reviewed the signs and symptoms recorded in the memory aid with each participant and parent/ guardian to confirm the severity and relationship to study vaccine and action taken (Supplemental Table 1). Unsolicited reactogenicity signs and symptoms were recorded as adverse events (AEs) and were monitored through day 29, whereas serious AEs (SAEs) were monitored and collected through day 181. No efficacy assessments were performed in the study. For serum antibody assays, blood was collected from all participants on days 1,11 , and $29( \pm 2)$ with additional blood draws on days $91( \pm 7)$ and $181( \pm 7)$ in cohort 1 .

A Safety Monitoring Committee (SMC), composed of two independent physicians and one independent statistician, conducted periodic reviews of all available, interim, blinded safety data every 6 months. Enrollment was to be stopped, and study medical monitor and SMC review performed before study restart for any death or SAE experienced by a participant, regardless of causality, or any severe or greater $A E$ assessed as possibly or probably related to the study vaccine.

Vaccine. Lyophilized PXVX0200 sachets stored at $-20^{\circ} \mathrm{C}$ were reconstituted in a sodium bicarbonate buffer, made using $100 \mathrm{~mL}$ of purified bottled water and single-dose buffer sachets containing $2.5 \mathrm{~g} \mathrm{NaHCO} 3,1.5 \mathrm{~g}$ ascorbic acid, and $0.2 \mathrm{~g}$ lactose. At reconstitution, the single-dose PXVX0200 solution contained $1 \times 10^{9} \mathrm{CFU}$ of live-attenuated $V$. cholerae. One vaccine lot, P700.610.00-7000005, was used in the study. Placebo was $100 \mathrm{~mL}$ of physiological $(0.9 \%)$ saline. Study vaccine was prepared and dispensed by an unblinded pharmacist to an unblinded dose administrator, and study product was consumed within 15 minutes of reconstitution. Participants received nothing by mouth for 60 minutes before and after vaccine administration. To maintain blinding, vaccine and placebo were dispensed in opaque cups and the unblinded pharmacist and dose administrator were not involved in postvaccination assessments and evaluations of PXVX0200 reactogenicity, AEs, acceptability, and palatability. The other study site personnel, the participants, their parents or guardians, and sponsor personnel did not know the participants' treatment assignments. The unblinded dose administrator had the option to add PureVia Stevia sweetener to the oral solution at the request of the parent and/or participant. The sweetener was intended to increase the palatability of the oral solution for pediatric study participants and to increase the likelihood that each participant would consume the full dose. Participants were monitored for acute reactions for 30 minutes after vaccination and were asked not to discuss the taste of the product with study staff or other study participants, as appropriate for age and cognitive level. Clinically significant findings immediately after vaccination were recorded as AEs. After completion of the 30-minute evaluation period, palatability was assessed by the participant and reviewed by blinded study staff using a 5-point hedonic scale. ${ }^{33}$ Blinding was maintained for each participant until they completed their day 181 assessment.

Immunology. To determine immunogenicity, titers of classic Inaba SVAs were measured at baseline before vaccine administration (day 1) and 10 and 28 days after vaccination (days 11 and 29) in all participants and also 90 and 180 days after vaccination (days 91 and 181) in cohort 1. These assays were performed at Q2 Solutions, San Juan Capistrano, CA, using assays with previously described methods transferred from the Applied Immunology Unit at the Center for Vaccine Development, University of Maryland, Baltimore. ${ }^{34}$

Statistical analysis. Sample size determination. The trial was designed to enable the independent evaluation of the immunogenicity of PXVX0200 in three separate age cohorts for two co-primary objectives: non-inferiority to adults in the rate of seroconversion and a minimum seroconversion rate of $70 \%$. The statistical Cls used for assessing the two primary objectives were adjusted for multiplicity by allocating $2 / 3$ of the type I error or alpha (0.033) to the non-inferiority objective and the remaining $1 / 3$ of the alpha $(0.0167)$ to the adequacy of the seroconversion rate. As a result, rather than the typical 95\% Cls, two-sided $96.7 \%$ Cls and $98.3 \%$ Cls for the noninferiority and adequacy endpoints, respectively, were used. 
The separate age cohorts were then tested sequentially within each objective to maintain the type 1 error across cohorts. The older age cohort (12-17 years) was assessed first, and, only if that age cohort achieved the success criterion, could the next younger age cohort be assessed.

To meet the co-primary endpoints, the lower bound of the two-sided $96.7 \% \mathrm{Cls}$ on the difference in seroconversion rates between children and adults aged 18-45 years was required to be greater than -10 percentage points and the lower bound of the two-sided $98.3 \% \mathrm{Cl}$ on the proportion of vaccinees who seroconverted between days 1 and 11 was required to be equal or exceed $70 \%$.

Assuming that the true seroconversion rate was $92.4 \%$ or higher, the sample size of 143 evaluable vaccinees for an age cohort afforded $93.3 \%$ power to demonstrate that the seroconversion rate within the age cohort was non-inferior to the 93.5\% rate observed in the 2,687 adult participants assessed in the lot consistency trial. ${ }^{28}$ Assuming that the rates in the three age cohorts are independent of one another, the overall power for demonstrating the non-inferiority of all three age cohorts $\left[(93.3 \%)^{3}\right]$ is $81 \%$.

Under the same assumptions as earlier-in particular, assuming the true seroconversion rate within an age cohort is at least $92.4 \%-143$ evaluable vaccinees provided greater than $99.9 \%$ power to establish that the lower bound on the cohortspecific rate is at least $70 \%$. Power would still be greater than $99.9 \%$ when requiring the lower bound on all three cohorts to be $70 \%$ or greater.

The overall power of meeting both primary objectives in all three age cohorts can be approximated by multiplying the power of meeting the non-inferiority objective by the power of meeting the $70 \%$ lower bound objective: $81 \% \times 99.9 \% \approx 81 \%$.

Immunogenicity. The primary endpoint was the proportion of participants achieving seroconversion, defined as a 4-fold or greater rise over day 1 SVAs, at day 11 after one dose of PXVX0200 in the immunogenicity evaluable population (IEP), which included the set of participants who received vaccination, consumed at least $80 \%$ of the study treatment, had evaluable results from both day 1 and day 11 , and had no major protocol violations that would affect immunogenicity. The proportion of seroconverters was called the seroconversion rate. Each rate was accompanied by a Cl calculated using the Wilson method, with the size of the $\mathrm{Cl}$ adjusted for the type of comparison needed (the placebo comparison used a $95 \%$ Cl). ${ }^{35}$ Cumulative seroconversion through a particular visit was defined as the occurrence of seroconversion at or before that visit. For each cohort, the proportion of vaccinated participants who experienced a 4-fold or greater increase in serum vibriocidal titer between day 1 and day 11 was calculated and the $98.3 \% \mathrm{Cls}$ on this proportion of seroconverters was calculated. The seroconversion rate for each cohort was compared with the seroconversion rate for the bridging population, vaccinees aged 18-45 years who participated in the lot consistency study, by calculating the difference between the two rates and computing a $96.7 \% \mathrm{Cl}$ for this difference. ${ }^{28}$

Additional immunogenicity objectives. The point estimate of the seroconversion rate for vaccinees as well as the cumulative seroconversion rate, and $95 \%$ Cls were also computed at the time points on days 29, 91, and 181 (day 29 only for cohort 2). The GMT and $95 \%$ Cls were computed for days 1, 11, and 29 (both cohorts), and for days 91 and 181 (cohort 1 only), and the geometric mean fold increase (GMFI) in titer in each treatment group was calculated from day 1 for each of the time point. GMTs and associated $95 \%$ Cls were calculated by first constructing the mean and $\mathrm{Cl}$ on log-transformed data and then back-transforming to the original data space.

Acceptability and palatability. Acceptability was evaluated by assessing the percent of participants in each age cohort able to complete the dosing according to the protocol. This was defined as the entire volume of dose being consumed within 15 minutes after reconstitution. Palatability of vaccine was assessed by the participants using a 5-point hedonic scale. ${ }^{33}$

Reactogenicity and safety. Solicited reactogenicity was summarized by the frequency in each treatment group of each of the solicited reactogenicity signs or symptoms as well as summaries by severity and treatment group and were compared using Fisher's exact tests (Supplemental Table 1). Unsolicited treatment emergent AEs and SAEs were summarized by system organ class and preferred term, by severity and by count and percent for PXVX0200 and placebo groups, by relationship to study vaccine, by age cohort, and overall.

\section{RESULTS}

Demographics. A total of 387 participants between the ages of 6 and 17 years were screened, and 374 were randomized across seven study sites, with 40 or more subjects enrolled at six sites. In cohort 1 (aged 12-17 years), 197 participants were screened, 189 were randomized, and 181 (95.8\%) completed the study through the day 181 visit. In cohort 2 (aged 6-11 years), 190 participants were screened, 185 were randomized, and $170(91.9 \%)$ completed the study through the day 181 visit (Figure 1). There were six participants who were randomized and had duplicate enrollment in error. These subjects were excluded from the immunogenicity evaluations but were included in the safety population. Participant characteristics for the study groups for randomized participants are presented in Table 1. Other than age, the demographic and other baseline characteristics of the treatment groups and cohorts were similar.

Immunogenicity. Non-inferiority. The day 11 seroconversion rates of the PXVX0200 participants in the IEP group compared with those in the adult bridging population from the lot consistency study are shown in Table 2 . For cohort 1, both primary objectives were met. Cohort 1 was non-inferior to the adult bridging population, with the lower limit of the $96.7 \% \mathrm{Cls}$ on the difference between the groups exceeding the required -10 percentage points (difference $=+5.8 \% ; 96.7 \%$ $\mathrm{Cl}:[2.4,7.1])$. There was also a statistically significant increase in the percentage of participants who seroconverted compared with the adult bridging population $(P<0.01)$. For the second co-primary endpoint, the cohort 1 participants had a 99.4\% day 11 seroconversion rate and the $98.3 \% \mathrm{Cl}$ was [95.4, 99.9\%], which well surpassed the protocol required lower confidence limit on seroconversion of $70 \%$.

For cohort 2, both co-primary objectives were also met. Cohort 2 was non-inferior to the adult bridging population, with the lower limit of the $96.7 \% \mathrm{Cl}$ on the difference between the groups exceeding the required -10 percentage points (difference $=+4.3 \%$; $96.7 \% \mathrm{Cl}$ : $[-0.3,6.2]$ ). For the second coprimary objective, the cohort 2 participants had a $97.8 \%$ day 11 seroconversion rate and the $98.3 \% \mathrm{Cl}$ was [92.5, 99.4\%], which was well greater than the protocol required lower limit of $70 \%$ with seroconversion. 


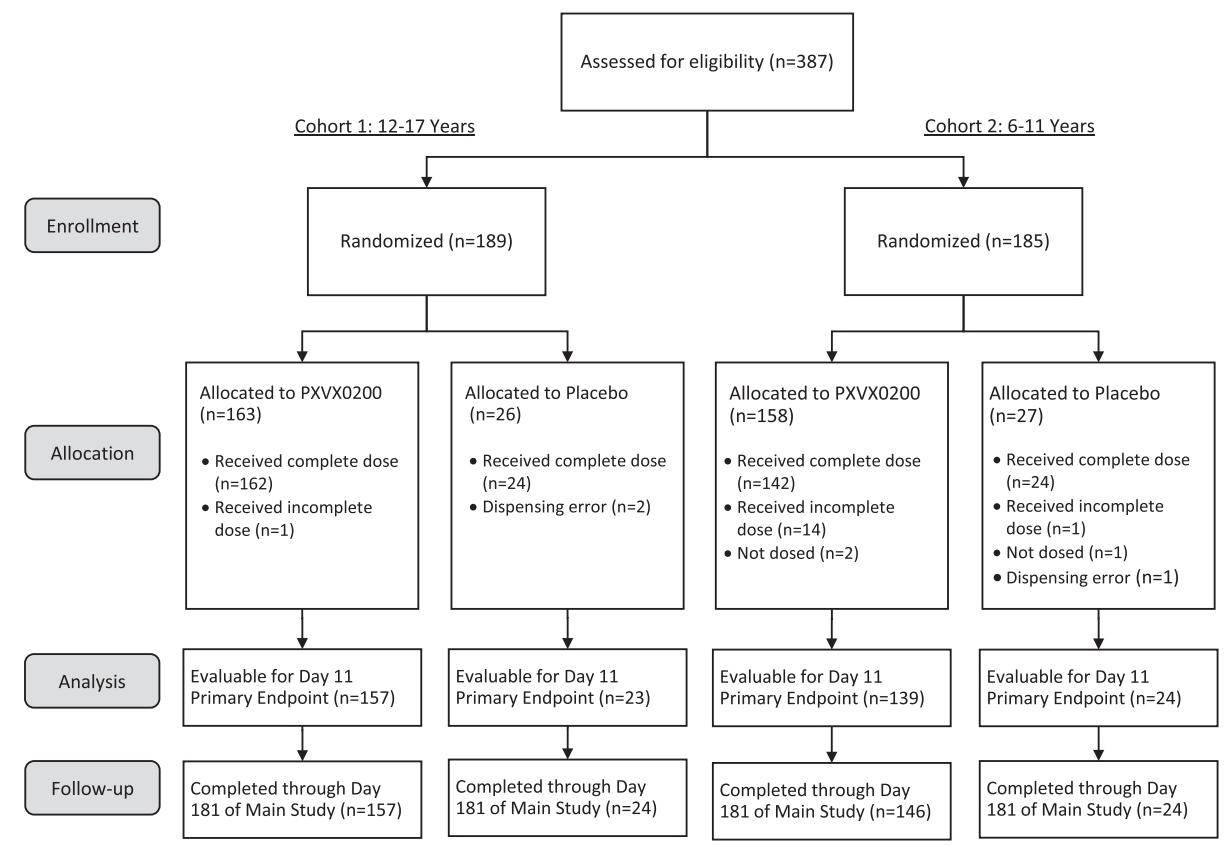

FIGURE 1. Participant disposition. Consolidated Standards of Reporting Tools (CONSORT) diagram for pediatric trial: Reasons for withdrawal were as follows: 10 participants withdrew consent, 10 were lost to follow-up, two were noncompliant with the protocol, and one ineligible participant was accidentally randomized and withdrawn.

The overall seroconversion rate was $98.6 \%$ among the pediatric PXVX0200 recipients (98.3\% Cl: [95.9\%, 99.6\%]) and was significantly higher than the adult seroconversion rate of $93.5 \%(P<0.01)$.
The primary dataset used for analysis was the IEP. However, a robustness analysis based on the modified intent-totreat population, which only excluded participants who were missing day 1 or day 11 SVA results, was included to

TABLE 1

Participant demographics by age, cohort, and treatment group

\begin{tabular}{|c|c|c|c|c|c|c|c|}
\hline \multirow{2}{*}{$\begin{array}{c}\text { Baseline } \\
\text { characteristic }\end{array}$} & \multicolumn{2}{|c|}{ Cohort 1 (aged 12-17 years) } & \multicolumn{2}{|c|}{ Cohort 2 (aged 6-11 years) } & \multicolumn{2}{|c|}{ Overall (aged 6-17 years) } & \multirow[b]{2}{*}{ Total $(N=374)$} \\
\hline & PXVX0200 $(N=163)$ & Placebo $(N=26)$ & PXVX0200 $(N=158)$ & Placebo $(N=27)$ & PXVX0200 $(N=321)$ & Placebo $(N=53)$ & \\
\hline \multicolumn{8}{|l|}{ Age (years) } \\
\hline Mean \pm SD & $14.4 \pm 1.7$ & $14.3 \pm 1.7$ & $8.6 \pm 1.8$ & $8.7 \pm 1.5$ & $11.5 \pm 3.4$ & $11.4 \pm 3.2$ & $11.5 \pm 3.4$ \\
\hline $\begin{array}{l}\text { Median } \\
\quad(\text { min-max })\end{array}$ & $14.0(12-17)$ & $15.0(12-17)$ & $9.0(6-11)$ & $9.0(6-11)$ & $12.0(6-17)$ & $11.0(6-17)$ & $12.0(6-17)$ \\
\hline \multicolumn{8}{|l|}{ Gender } \\
\hline Male, $n(\%)$ & $88(54.0)$ & $14(53.8)$ & 77 (48.7) & 17 (63.0) & $165(51.4)$ & $31(58.5)$ & $196(52.4)$ \\
\hline Female, $n(\%)$ & 75 (46.0) & $12(46.2)$ & 81 (51.3) & $10(37.0)$ & 156 (48.6) & $22(41.5)$ & $178(47.6)$ \\
\hline \multicolumn{8}{|l|}{ Race } \\
\hline $\begin{array}{l}\text { American Indian } \\
\text { or Alaskan } \\
\text { Native, } n(\%)\end{array}$ & 0 & $1(3.8)$ & 0 & $1(3.7)$ & 0 & $2(3.8)$ & $2(0.5)$ \\
\hline Asian, $n(\%)$ & $1(0.6)$ & 0 & $4(2.5)$ & 0 & $5(1.6)$ & 0 & $5(1.3)$ \\
\hline $\begin{array}{l}\text { Native Hawaiian } \\
\text { or other Pacific } \\
\text { Islander }\end{array}$ & 0 & 0 & 0 & 0 & 0 & 0 & 0 \\
\hline $\begin{array}{l}\text { Black or African } \\
\text { American, } n \\
(\%)\end{array}$ & $28(17.2)$ & $4(15.4)$ & $53(33.5)$ & 5 (18.5) & 81 (25.2) & $9(17.0)$ & $90(24.1)$ \\
\hline White, $n(\%)$ & $121(74.2)$ & $21(80.8)$ & $86(54.4)$ & $18(66.7)$ & 207 (64.5) & 39 (73.6) & $246(65.8)$ \\
\hline Multiple, $n(\%)$ & $13(8.0)$ & 0 & $15(9.5)$ & $3(11.1)$ & $28(8.7)$ & $3(5.7)$ & $31(8.3)$ \\
\hline Other & 0 & 0 & 0 & 0 & 0 & 0 & 0 \\
\hline \multicolumn{8}{|l|}{ Ethnicity } \\
\hline $\begin{array}{l}\text { Hispanic or } \\
\quad \text { Latino, } n(\%)\end{array}$ & $18(11.0)$ & 7 (26.9) & $11(7.0)$ & $2(7.4)$ & $29(9.0)$ & $9(17.0)$ & 38 (10.2) \\
\hline $\begin{array}{l}\text { Not Hispanic or } \\
\text { Latino, } n(\%)\end{array}$ & $145(89.0)$ & 19 (73.1) & $147(93.0)$ & $25(92.6)$ & $292(91.0)$ & $44(83.0)$ & $336(89.8)$ \\
\hline \multicolumn{8}{|l|}{ BMI $\left(\mathrm{kg} / \mathrm{m}^{2}\right)$} \\
\hline Mean (SD) & $23.6(5.7)$ & $25.0(4.8)$ & $18.7(5.2)$ & $17.5(3.1)$ & $21.2(6.0)$ & $21.2(5.5)$ & $21.2(5.9)$ \\
\hline $\begin{array}{l}\text { Median } \\
\quad(\min -\max )\end{array}$ & $22.5(14.3-45.1)$ & $24.2(17.8-36.6)$ & $17.1(11.7-44.6)$ & $16.9(13.0-26.2)$ & $20.4(11.7-45.1)$ & $20.0(13.0-36.6)$ & $20.3(11.7-45.1)$ \\
\hline
\end{tabular}


TABLE 2

Comparison of seroconversion rates at day 11 visit by age-group compared with the adult bridging population

\begin{tabular}{|c|c|c|c|c|}
\hline & $\begin{array}{c}\text { Adults } \\
\text { (aged } 18-45 \text { years) }\end{array}$ & $\begin{array}{l}\text { Cohort } 1 \\
\text { (aged 12-17 years) }\end{array}$ & $\begin{array}{l}\text { Cohort } 2 \\
\text { (aged 6-11 years) }\end{array}$ & $\begin{array}{c}\text { Overall } \\
\text { (aged 6-17 years) }\end{array}$ \\
\hline Seroconversion & PXVX0200 & PXVX0200 & PXVX0200 & PXVX0200 \\
\hline \multicolumn{5}{|l|}{ Day 11 visit } \\
\hline$N$ analyzable & 2,687 & 157 & 139 & 296 \\
\hline$N(\%)$ seroconverted $[98.3 \% \mathrm{Cl}]$ & $\begin{array}{c}2,513(93.5) \\
{[92.3 \%, 94.6 \%]}\end{array}$ & $\begin{array}{c}156(99.4) \\
{[95.4 \%, 99.9 \%}\end{array}$ & $\begin{array}{c}136(97.8) \\
{[92.5 \%, 99.4 \%]}\end{array}$ & $\begin{array}{c}292(98.6) \\
{[95.9 \% .99 .6 \%]}\end{array}$ \\
\hline Difference (Cohort minus adults) & - & $5.8 \%$ & $4.3 \%$ & $5.1 \%$ \\
\hline $96.7 \% \mathrm{Cl}$ on \% difference & - & {$[2.4 \%, 7.1 \%]$} & {$[-0.3 \%, 6.2 \%]$} & {$[2.6 \%, 6.5 \%]$} \\
\hline$P$-value & - & 0.0009 & 0.0455 & $<0.0001$ \\
\hline
\end{tabular}

determine the degree to which protocol deviations may have affected the results. The overall conclusion of non-inferiority to the adults and seroconversion was unaffected by the choice of analysis population.

Additional immunogenicity objectives. The cumulative seroconversion rates by the treatment group and cohort are shown in Table 3. The cumulative seroconversion rate at day 29 for PXVX0200 recipients in cohort 1 was $100 \%$ versus $0 \%$ for the placebo recipients $(P<0.0001)$. The cumulative seroconversion rate at day 29 for cohort 2 was $97.8 \%$ versus $8.3 \%$ for the placebo recipients $(P<0.0001)$. The overall day 29 cumulative seroconversion rate was $99.0 \%$ for PXVX0200 recipients versus $4.3 \%$ for placebo recipients $(P<0.0001)$.

The GMT of serum vibriocidal titers for PXVX0200 recipients in cohort 1 peaked at 8,735 on day 11 and decreased through day 181 at which point titers remained significantly greater than placebo (Table 4). The GMT of serum vibriocidal titers for PXVX0200 recipients in cohort 2 peaked at 8,305 on day 11 and decreased to 1952 at day 29. In both cohorts, all comparisons of PXVX0200 to placebo were highly significant $(P<$ 0.0001). Figure 2 shows GMTs over time for vaccine recipients along with the analogous measurements from the bridging population in the adult lot consistency study.

The results for GMFI in SVA titers are shown in Table 5. The GMFI of vibriocidal antibodies for cohort 1 PXVX0200 participants was 272 times baseline value at day 11 and 85 times baseline at day 29. The GMFI of vibriocidal antibodies for cohort 2 PXVX0200 participants was 264 times baseline at day 11 and 62 times baseline at day 29. As with the GMTs, the comparisons of PXVX0200 to placebo for mean fold increase were highly significant for both cohorts at all visits $(P<0.0001)$.

Acceptability. In cohort 1, 99.4\% of PXVX0200 recipients and $100 \%$ of placebo recipients were completely dosed with the correct respective oral solutions (Table 6). In cohort 2, 91.0\% of PXVX0200 recipients and $96.2 \%$ of placebo recipients were completely dosed with the correct respective oral solutions.

Palatability. Results of palatability testing are presented in Table 7 . The palatability assessments did not differ significantly between the vaccine and placebo groups within each cohort. Issues with palatability possibly contributed to the percentage of participants who did not consume a complete dose of their respective randomized treatment in cohort 2. Of cohort 2 participants with unacceptable dosing, palatability was reported as "very bad" by $80 \%$ of PVXV0200 recipients and by one placebo recipient. Addition of PureVia Stevia sweetener did not improve participants' opinions regarding treatment palatability.

Reactogenicity and safety. The frequency and severity of solicited reactogenicity signs and symptoms for PXVX0200 and placebo recipients are reported in Table 8 . Reactogenicity was more common in cohort 1 than in cohort 2. The number of participants with any solicited AE was similar between both treatment groups, and the median duration of symptoms was

TABLE 3

Cumulative seroconversion rate by treatment group and cohort

\begin{tabular}{|c|c|c|c|c|c|c|}
\hline \multirow[b]{2}{*}{ Seroconversion } & \multicolumn{2}{|c|}{ Cohort 1 (aged 12-17 years) } & \multicolumn{2}{|c|}{ Cohort 2 (aged 6-11 years) } & \multicolumn{2}{|c|}{ Overall (aged 6-17 years) } \\
\hline & $\begin{array}{l}P X V X 0200 \\
(N=157)\end{array}$ & $\begin{array}{l}\text { Placebo } \\
(N=23)\end{array}$ & $\begin{array}{l}P X V X 0200 \\
(N=139)\end{array}$ & $\begin{array}{l}\text { Placebo } \\
(N=24)\end{array}$ & $\begin{array}{l}P X V X 0200 \\
(N=296)\end{array}$ & $\begin{array}{l}\text { Placebo } \\
(N=47)\end{array}$ \\
\hline \multicolumn{7}{|l|}{ Day 11 visit } \\
\hline$N$ analyzable & 157 & 23 & 139 & 24 & 296 & 47 \\
\hline $\begin{array}{l}N(\%) \text { seroconverted } \\
(95 \% \mathrm{Cl})\end{array}$ & $\begin{array}{c}156(99.4 \%)^{\star} \\
{[96.5 \%, 99.9 \%]}\end{array}$ & $\begin{array}{l}0[0 \%, \\
14.3 \%]\end{array}$ & $\begin{array}{c}136(97.8 \%)^{*} \\
{[93.8 \%, 99.3 \%]}\end{array}$ & $\begin{array}{c}1(4.2 \%) \\
{[0.7 \%, 20.2 \%]}\end{array}$ & $\begin{array}{c}292(98.6 \%)^{\star} \\
{[96.6 \%, 99.5 \%]}\end{array}$ & $\begin{array}{c}1(2.1 \%) \\
{[0.4 \%, 11.1 \%]}\end{array}$ \\
\hline \multicolumn{7}{|l|}{ Day 29 visit } \\
\hline$N$ analyzable & 157 & 23 & 139 & 24 & 296 & 47 \\
\hline$N$ (\%) seroconverted & $157(100 \%)^{\star}$ & $0[0 \%$, & $136(97.8 \%)^{\star}$ & $2(8.3 \%)$ & $293(99.0 \%)^{\star}$ & $2(4.3 \%)$ \\
\hline \multicolumn{7}{|l|}{ Day 91 visit } \\
\hline$N$ analyzable & 157 & 23 & - & - & - & - \\
\hline $\begin{array}{l}N(\%) \text { seroconverted } \\
(95 \% \mathrm{Cl})\end{array}$ & $\begin{array}{c}157(100 \%)^{\star} \\
{[97.6 \%, 100 \%]}\end{array}$ & $\begin{array}{l}0[0 \%, \\
14.3 \%]\end{array}$ & - & - & - & - \\
\hline \multicolumn{7}{|l|}{ Day 181 visit } \\
\hline$N$ analyzable & 157 & 23 & - & - & - & - \\
\hline $\begin{array}{l}N(\%) \text { seroconverted } \\
(95 \% \mathrm{Cl})\end{array}$ & $\begin{array}{c}157(100 \%)^{\star} \\
{[97.6 \%, 100 \%]}\end{array}$ & $\begin{array}{l}0[0 \%, \\
14.3 \%]\end{array}$ & - & - & - & - \\
\hline
\end{tabular}


TABLE 4

Geometric mean titers (GMT) against classical Inaba Vibrio cholerae, all time points by age-group and cohort, immunogenicity evaluable population

\begin{tabular}{|c|c|c|c|c|c|c|}
\hline \multirow[b]{2}{*}{ Geometric mean } & \multicolumn{2}{|c|}{ Cohort 1 (aged 12-17 years) } & \multicolumn{2}{|c|}{ Cohort 2 (aged 6-11 years) } & \multicolumn{2}{|c|}{ Overall (aged 6-17 years) } \\
\hline & PXVX0200 $(N=157)$ & Placebo $(N=23)$ & PXVX0200 $(N=139)$ & Placebo $(N=24)$ & PXVX0200 (N = 296) & Placebo $(N=47)$ \\
\hline \multicolumn{7}{|l|}{ Day 1 visit } \\
\hline$N$ analyzable & 157 & 23 & 139 & 24 & 296 & 47 \\
\hline GMT & 32 & 44 & 32 & 36 & 32 & 39 \\
\hline (95\% Cl) & {$[28,37]$} & {$[28,70]$} & {$[28,36]$} & {$[24,52]$} & {$[29,35]$} & {$[30,53]$} \\
\hline Min, max & 20,640 & $20,1,280$ & $20,1,280$ & 20,640 & $20,1,280$ & $20,1,280$ \\
\hline \multicolumn{7}{|l|}{ Day 11 visit } \\
\hline$N$ analyzable & 157 & 23 & 139 & 24 & 296 & 47 \\
\hline GMT & $8,735^{\star}$ & 41 & $8,305^{\star}$ & 40 & $8,531^{*}$ & 41 \\
\hline (95\% Cl) & {$[7,053,10,819]$} & {$[26,65]$} & {$[6,516,10,586]$} & {$[23,70]$} & {$[7,270,10,009]$,} & {$[29,58]$} \\
\hline Min, $\max$ & $20,81,920$ & $20,1,280$ & $40,163,840$ & $20,5,120$ & $20,163,840$ & $20,5,120$ \\
\hline \multicolumn{7}{|l|}{ Day 29 visit } \\
\hline$N$ analyzable & 156 & 23 & 138 & 23 & 294 & 46 \\
\hline GMT & $2,748^{*}$ & 43 & $1,952^{\star}$ & 40 & $2,341^{\star}$ & 41 \\
\hline$(95 \% \mathrm{Cl})$ & {$[2,311,3,270]$} & {$[27,67]$} & {$[1,554,2,452]$} & {$[22,72]$} & {$[2,031,2,697]$} & {$[29,59]$} \\
\hline Min, $\max$ & $320,20,480$ & 20,640 & $40,40,960$ & $20,5,120$ & $40,40,960$ & $20,5,120$ \\
\hline \multicolumn{7}{|l|}{ Day 91 visit } \\
\hline$N$ analyzable & 153 & 23 & - & - & - & - \\
\hline GMT & $319^{\star}$ & 43 & - & - & - & - \\
\hline (95\% Cl) & {$[263,386]$} & {$[29,63]$} & - & - & - & - \\
\hline Min, max & $20,5,120$ & 20,640 & - & - & - & - \\
\hline \multicolumn{7}{|l|}{ Day 181 visit } \\
\hline$N$ analyzable & 151 & 21 & - & - & - & - \\
\hline GMT & $186^{\star}$ & 39 & - & - & - & - \\
\hline$(95 \% \mathrm{Cl})$ & {$[154,225]$} & {$[26,58]$} & - & - & - & - \\
\hline Min, $\max$ & $20,5,120$ & 20,320 & - & - & - & - \\
\hline
\end{tabular}

3 days for cohort 1 and 2 days for cohort 2. Among solicited AEs, the most frequent across both cohorts and treatment groups were tiredness, headache, and abdominal pain. Diarrhea was reported in 6/165 (3.6\%) of PXVX0200 recipients in cohort 1 and was not reported by any study participants in cohort 2. Abdominal pain and vomiting were more frequently reported by PXVX0200 recipients than by placebo recipients, but these differences were not statistically significant. Across both cohorts, there were four participants who reported severe solicited AEs that were considered treatment related, including one with nausea, vomiting, and diarrhea; one with abdominal pain and diarrhea; and one each with fever or diarrhea only. All of these participants were PXVX0200 recipients. Study enrollment was stopped five times for severe

Geometric Mean Titer $(95 \% \mathrm{Cl})$ of Serum Vibriocidal Antibody

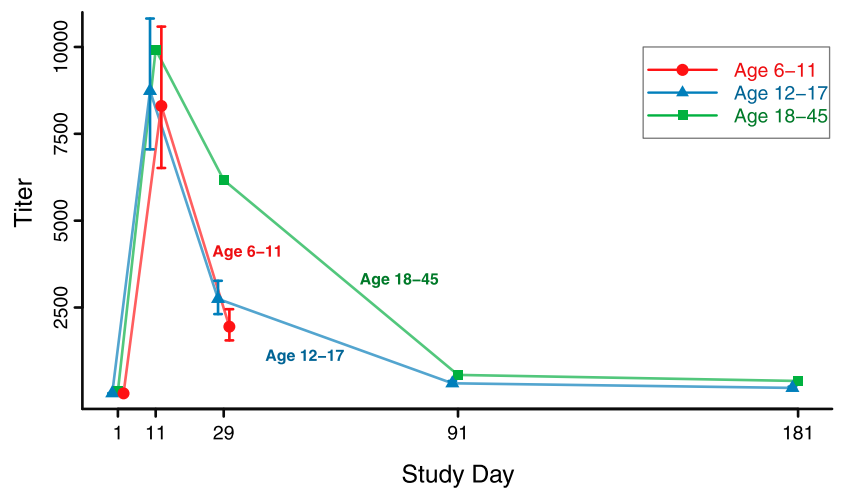

FIGURE 2. Geometric mean titer evels by age cohort and treatment group. Cohort 2 (aged 6-11 years) titers followed to day 29 only. This figure appears in color at www.ajtmh.org. solicited reactogenicity signs/symptoms, four considered possibly related to study product by the investigator, one severe fever considered unrelated, and for one unrelated SAE. In each case, SMC review was performed and the study restarted without modification. Unsolicited AEs were reported by $24.5 \%$ of PXVX0200 recipients and $32.7 \%$ of placebo recipients, and most were considered unrelated to study treatment. There were no deaths, treatment-related SAEs, or discontinuations due to AEs in either age cohort or treatment group. There was one unrelated SAE, a lower limb fracture, reported in a PXVX0200 recipient.

\section{DISCUSSION}

Cholera is a potentially lethal infection that occurs throughout the world in both endemic forms and as explosive outbreaks in "virgin soil" populations. ${ }^{2,3}$ It also represents a threat to both children and adults from developed countries who travel to cholera-endemic areas and can cause severe and fatal disease. ${ }^{4,6-8,36,37}$ A single-dose vaccine that could provide rapid protection in all age-groups to both travelers to and inhabitants of countries at risk for cholera would be ideal.

This study documents the safety, immunogenicity, and tolerability of a single-dose, live oral cholera vaccine PXVX0200 in children and adolescents aged 6-17 years in a developed country. Because $V$. cholerae challenge studies are not feasible in a pediatric population, we undertook an immunologic bridging study with SVA seroconversion as a correlate of protection. Bridging studies are performed to extrapolate efficacy for vaccines for which a correlate of protection exists and SVA seroconversion, defined as $a \geq 4$-fold rise in vibriocidal antibody, was shown to be a strong serological correlate of protection in the PXVX0200 challenge study. ${ }^{26,27,38}$ In the current trial, SVA seroconversion 10 days 
TABLE 5

Geometric mean fold increase against classical Inaba Vibrio cholerae all time points by age group and cohort-immunogenicity evaluable population

\begin{tabular}{|c|c|c|c|c|c|c|}
\hline \multirow[b]{2}{*}{ Fold increase } & \multicolumn{2}{|c|}{ Cohort 1 (aged 12-17 years) } & \multicolumn{2}{|c|}{ Cohort 2 (aged 6-11 years) } & \multicolumn{2}{|c|}{ Overall (aged 6-17 years) } \\
\hline & PXVX0200 $(N=160)$ & Placebo $(N=24)$ & $\operatorname{PXVX0200}(N=148)$ & Placebo $(N=24)$ & PXVX0200 (N=308) & Placebo $(N=48)$ \\
\hline \multicolumn{7}{|l|}{ Day 11 visit } \\
\hline$N$ analyzable & 157 & 23 & 139 & 24 & 296 & 47 \\
\hline Mean fold increase & $272^{*}$ & 1 & $264^{\star}$ & 1 & $268^{\star}$ & 1 \\
\hline$(95 \% \mathrm{Cl})$ & {$[222,334]$} & {$[1,1]$} & {$[204,341]$} & {$[1,2]$} & {$[229,315]$} & {$[1,1]$} \\
\hline Min, max & $1,4,096$ & $0.3,2$ & $1,4,096$ & 1,256 & $1,4,096$ & $0.3,256$ \\
\hline \multicolumn{7}{|l|}{ Day 29 visit } \\
\hline$N$ analyzable & 156 & 23 & 138 & 23 & 294 & 46 \\
\hline Mean fold increase & $85^{\star}$ & 1 & $62^{\star}$ & 1 & $73^{\star}$ & 1 \\
\hline$(95 \% \mathrm{Cl})$ & {$[72,102]$} & {$[1,1]$} & {$[49,78]$} & {$[1,2]$} & {$[64,85]$} & {$[1,1]$} \\
\hline Min, max & $8,1,024$ & $0.3,2$ & $1,1,024$ & 1,32 & $1,1,024$ & $0.3,32$ \\
\hline \multicolumn{7}{|l|}{ Day 91 visit } \\
\hline$N$ analyzable & 153 & 23 & - & - & - & - \\
\hline Mean fold increase & $10^{*}$ & 1 & - & - & - & - \\
\hline$(95 \% \mathrm{Cl})$ & {$[8,12]$} & {$[1,1]$} & - & - & - & - \\
\hline Min, max & 1,256 & $0.1,2$ & - & - & - & - \\
\hline \multicolumn{7}{|l|}{ Day 181 visit } \\
\hline$N$ analyzable & 151 & 21 & - & - & - & - \\
\hline Mean fold increase & $6^{*}$ & 1 & - & - & - & - \\
\hline$(95 \% \mathrm{Cl})$ & {$[5,7]$} & {$[1,1]$} & - & - & - & - \\
\hline Min, max & 1,128 & $0.3,2$ & - & - & - & - \\
\hline
\end{tabular}

after vaccination occurred in $99.4 \%$ of recipients aged $12-17$ years (cohort 1) and in $97.8 \%$ of vaccine recipients aged $6-11$ years (cohort 2) versus $0 \%$ and $4.2 \%$, respectively, in placebo recipients $(P<0.0001)$. These rates compared favorably with the seroconversion rate of $93.5 \%$ seen in the bridging population of adults aged 18-45 years in the phase 3 lot consistency study, and because seroconversion was greater than $70 \%$ in each cohort, both co-primary endpoints were met. Of note, although the SVA seroconversion rate was non-inferior in participants aged 6-11 years when compared with adults aged 18-45 years, it was significantly higher than that for adults in participants aged $12-17$ years $(P=0.0009)$. Because SVAs have long been known to be a correlate of protection against cholera infection, vaccine recipients aged $6-17$ years would be predicted to be protected against cholera-induced diarrhea. $^{26,27,39,40}$ The day 11 SVA seroconversion rates in placebo recipients aged $6-17$ years $(2.1 \%)$ were also similar to those seen in adult placebo recipients aged 18-45 years $(4.2 \%)$.

Studies of CVD 103-HgR in North American adults also documented protection against diarrhea caused by both the classical and El Tor biotypes and both the Inaba and Ogawa serotypes of $V$. cholerae 01 , and protection against these other types of cholera in children and adolescents could possibly be inferred. ${ }^{41-44}$ GMT kinetics in cohort 1 and cohort 2 (Figure 2) were similar to those in adults aged $18-45$ years in the lot consistency study. ${ }^{28}$ Thus, analysis of GMTs, geometric fold increase, and cumulative seroconversion rates confirm that PXVX0200 was immunogenic in this pediatric population.

Previous studies of a single dose of the CVD 103-HgR vaccine strain in children in developing countries showed that the vaccine was well tolerated and demonstrated that the immune responses, as measured by SVA seroconversion rates and GMTs, were lower than the responses seen in adults in developed countries, as well as in Thai adults from higher socioeconomic levels. ${ }^{14-17,19-23,44-47}$ As a result, CVD 103$\mathrm{HgR}$ dosages were increased 10-fold to achieve higher rates of seroconversion and the vaccine was marketed in developing countries as the Orochol $E$ formulation which contained $5 \times 10^{9} \mathrm{CFU} /$ dose. For example, Suharyono documented SVA seroconversion in $16 \%$ of 5 - to 9 -year-old participants living in squalid conditions in Indonesia immunized with $5 \times$ $10^{8} \mathrm{CFU}$ of CVD 103-HgR and in $75-87 \%$ of those immunized with $5 \times 10^{9} \mathrm{CFU}^{14}$ Most of the previous studies of CVD 103$\mathrm{HgR}$ in children in developing countries used a dose of $5 \times 10^{9}$ CFU with seroconversion seen in only $22-78 \%$ of vaccine recipients.

Immune responses to oral vaccines, including polio virus, rotavirus, and cholera, may be lower in infants and children in developing countries, and thus higher CVD 103-HgR dosages are required to induce protective antibodies. ${ }^{30-32}$ These reduced immune responses may be due to multiple factors, including malnutrition, maternal undernutrition during pregnancy, small bowel overgrowth with intestinal mucosa damage (chronic environmental enteropathy) from living in fecally

TABLE 6

Vaccine acceptability by age cohort and randomized treatment group

\begin{tabular}{|c|c|c|c|c|c|c|}
\hline \multirow[b]{2}{*}{ Dosing } & \multicolumn{2}{|c|}{ Cohort 1 (aged $12-17$ years) } & \multicolumn{2}{|c|}{ Cohort 2 (aged 6-11 years) } & \multicolumn{2}{|c|}{ Overall (aged 6-17 years) } \\
\hline & $\operatorname{PXVX0200}(N=163)$ & Placebo $(N=26)$ & $\operatorname{PXVX0200}(N=156)$ & Placebo $(N=26)$ & PXVX0200 (N = 319) & Placebo $(N=52)$ \\
\hline \multicolumn{7}{|c|}{ Complete dose consumed } \\
\hline Yes, $n(\%)$ & $162(99.4)$ & $26(100)$ & $142(91.0)$ & $25(96.2)$ & 304 (95.3) & $51(98.1)$ \\
\hline No, $n(\%)$ & $1(0.6)$ & 0 & $14(9.0)$ & $1(3.8)$ & $15(4.7)$ & $1(1.9)$ \\
\hline
\end{tabular}


TABLE 7

Palatability by age cohort and treatment group in subjects who received treatment

\begin{tabular}{|c|c|c|c|c|c|c|}
\hline \multirow[b]{2}{*}{ Response ( $n[\%])$} & \multicolumn{2}{|c|}{ Cohort 1 (aged 12-17 years) } & \multicolumn{2}{|c|}{ Cohort 2 (aged 6-11 years) } & \multicolumn{2}{|c|}{ Overall (aged 6-17 years) } \\
\hline & $\operatorname{PXVX0200}(\mathrm{N}=163)$ & Placebo $(\mathrm{N}=26)$ & PXVX0200 (N = 156) & Placebo $(\mathrm{N}=26)$ & PXVX0200 (N = 319) & Placebo $(N=52)$ \\
\hline \multicolumn{7}{|l|}{ Palatability rating } \\
\hline Very bad & $12(7.4)$ & $1(3.8)$ & 34 (21.8) & $6(23.1)$ & $46(14.4)$ & 7 (13.5) \\
\hline $\mathrm{Bad}$ & $50(30.7)$ & 7 (26.9) & 30 (19.2) & $6(23.1)$ & $80(25.1)$ & $13(25.0)$ \\
\hline Neutral & 64 (39.3) & $10(38.5)$ & $37(23.7)$ & $10(38.5)$ & $101(31.7)$ & $20(38.5)$ \\
\hline Good & $29(17.8)$ & $6(23.1)$ & $27(17.3)$ & $1(3.8)$ & 56 (17.6) & 7 (13.5) \\
\hline Very good & $8(4.9)$ & $2(7.7)$ & $28(17.9)$ & $3(11.5)$ & $36(11.3)$ & $5(9.6)$ \\
\hline \multicolumn{7}{|l|}{ Sweetener added } \\
\hline Yes & 144 (88.3) & $22(84.6)$ & 149 (95.5) & 25 (96.2) & $293(91.8)$ & $47(90.4)$ \\
\hline
\end{tabular}

contaminated environments, parasitic infections, intestinal enteric virus infections, interference from breast milk antibody, and preexisting immunity due to natural exposure to infection. Chronic environmental enteropathy, also known as tropical enteropathy, a subclinical inflammatory condition of the gut, is associated with changes in the gut microbiota and intestinal barrier function, upregulation of pro-inflammatory cytokines and chemokines, and decreased responsiveness to oral polio and rotavirus vaccines. ${ }^{32}$ A study of small bowel overgrowth documented an association between peak breath hydrogen testing and diminished seroconversion following immunization with CVD 103-HgR. ${ }^{19}$ An efficacy trial of CVD 103-HgR in Indonesia documented seroconversion in $42 \%$ of participants with high baseline vibriocidal titers versus $86 \%$ in those with low titers, suggesting that some individuals may already be immune to cholera and that their serum titers are not boosted substantially by immunization. ${ }^{21,31}$ Another trial documented increased seroconversion following vaccination with CVD
103-HgR in children with ascariasis treated with albendazole. ${ }^{22}$ None of the conditions associated with decreased seroconversion described here is common in the United States or other developed countries, and it is expected that children from these countries will mount a more substantial immune response to PXVX0200.

The study vaccine was well accepted with $99.4 \%$ and $91.0 \%$, respectively, of cohort 1 and cohort 2 PXVX0200 recipients able to complete dosing, suggesting that age influenced the willingness and ability to consume the oral solution. It is likely that palatability impacted the acceptability of the study vaccine because $8 / 10$ (80\%) of the cohort 2 PXVX0200 recipients with unacceptable dosing rated palatability as "very bad." Overall, there was no significant difference in palatability assessments between vaccine and placebo recipients in each cohort. It is notable that in one trial of CVD 103-HgR performed in infants and toddlers in Chile, the seroconversion rate was similar in fully vaccinated participants $(66 \%)$ and in those who

TABLE 8

Frequency and severity of solicited reactogenicity reported

\begin{tabular}{|c|c|c|c|c|c|c|}
\hline \multirow[b]{2}{*}{ Solicited event [n (\%)] } & \multicolumn{2}{|c|}{ Cohort 1 (aged 12-17 years) } & \multicolumn{2}{|c|}{ Cohort 2 (aged 6-11 years) } & \multicolumn{2}{|c|}{ Overall (aged 6-17 years) } \\
\hline & $\operatorname{PXVX0200}(N=165)$ & Placebo $(N=24)$ & $\operatorname{PXVX0200}(N=157)$ & Placebo $(N=25)$ & PXVX0200 (N = 322) & Placebo $(N=49)$ \\
\hline Any event & $113(68.5)$ & $16(66.7)$ & $86(54.8)$ & $13(52.0)$ & 199 (61.8) & $29(59.2)$ \\
\hline Mild/moderate & $109(66.1)$ & 15 (62.5) & $81(51.6)$ & $13(52.0)$ & 190 (59.0) & $28(57.1)$ \\
\hline Severe & $4(2.4)^{\star}$ & $1(4.2)$ & $5(3.2)$ & 0 & $9(2.8)^{\star}$ & $1(2.0)$ \\
\hline Tiredness & $67(40.6)$ & $9(37.5)$ & 55 (35.0) & $8(32.0)$ & $122(37.9)$ & $17(34.7)$ \\
\hline Mild/moderate & $66(40.0)$ & 8 (33.3) & $54(34.4)$ & $8(32.0)$ & 120 (37.2) & $16(32.6)$ \\
\hline Severe & $1(0.6)^{\star}$ & $1(4.2)$ & $1(0.6)$ & 0 & $2(0.6)^{\star}$ & $1(2.0)$ \\
\hline Headache & $74(44.8)$ & $11(45.8)$ & $41(26.1)$ & $6(24.0)$ & $115(35.7)$ & $17(34.7)$ \\
\hline Mild/moderate & 73 (44.2) & $11(45.8)$ & $39(24.8)$ & $6(24.0)$ & $112(34.8)$ & $17(34.7)$ \\
\hline Severe & $1(0.6)$ & 0 & $2(1.3)$ & 0 & $3(0.9)$ & 0 \\
\hline Abdominal pain & $62(37.6)$ & $4(16.7)$ & $43(27.4)$ & $6(24.0)$ & 105 (32.6) & $10(20.4)$ \\
\hline Mild/moderate & $61(37.0)$ & $4(16.7)$ & $43(27.4)$ & $6(24.0)$ & 104 (32.3) & $10(20.4)$ \\
\hline Severe & $1(0.6)$ & 0 & 0 & 0 & $1(0.3)$ & 0 \\
\hline Lack of appetite & $48(29.1)$ & $3(12.5)$ & 24 (15.3) & $5(20.0)$ & $72(22.4)$ & 8 (16.3) \\
\hline Mild/moderate & $48(29.1)$ & $3(12.5)$ & 23 (14.6) & $5(20.0)$ & 71 (22.0) & 8 (16.3) \\
\hline Severe & 0 & 0 & $1(0.6)$ & 0 & $1(0.3)$ & 0 \\
\hline Nausea & $37(22.4)$ & $6(25.0)$ & $22(14.0)$ & $4(16.0)$ & 59 (18.3) & $10(20.4)$ \\
\hline Mild/moderate & $36(21.8)$ & $6(25.0)$ & $22(14.0)$ & $4(16.0)$ & $58(18.0)$ & $10(20.4)$ \\
\hline Severe & $1(0.6)$ & 0 & 0 & 0 & $1(0.3)$ & 0 \\
\hline Vomiting & $9(5.5)$ & 0 & $7(4.5)$ & 0 & $16(5.0)$ & 0 \\
\hline Mild/moderate & $8(4.8)$ & 0 & $7(4.5)$ & 0 & $15(4.7)$ & 0 \\
\hline Severe & $1(0.6)$ & 0 & 0 & 0 & $1(0.3)$ & 0 \\
\hline Fever & $3(1.8)$ & 0 & $5(3.2)$ & $1(4.0)$ & $8(2.5)$ & $1(2.0)$ \\
\hline Mild/moderate & $2(1.2)$ & 0 & $1(0.6)$ & $1(4.0)$ & $3(0.9)$ & $1(2.0)$ \\
\hline Severe & $1(0.6)$ & 0 & $4(2.5)$ & 0 & $5(1.6)$ & 0 \\
\hline Diarrhea & $6(3.6)$ & $1(4.2)$ & 0 & 0 & $6(1.9)$ & $1(2.0)$ \\
\hline Mild/moderate & $3(1.8)$ & $1(4.2)$ & 0 & 0 & $3(0.9)$ & $1(2.0)$ \\
\hline Severe & $3(1.8)$ & 0 & 0 & 0 & $3(0.9)$ & 0 \\
\hline
\end{tabular}

statistically significant.

* Includes one case of potentially life-threatening tiredness. 
ingested a smaller fraction of the vaccine (63\%). ${ }^{20}$ However, these results may not be applicable to the current study, and, in general, the acceptability and palatability of any pediatric oral medication or vaccine are critical to compliance and, thus, its effectiveness. ${ }^{48,49}$ Addition of sweetener did not improve palatability and will not be used in future PXVX0200 pediatric trials.

Other than one small study performed in Austria, there are no previous studies of the safety of CVD 103-HgR in children in developed countries. ${ }^{18}$ The data from this study demonstrated that PXVX0200 is well tolerated in children and adolescents, with a safety profile similar to that seen in adults and also to Orochol, the previously marketed form of CVD 103$\mathrm{HgR}^{24-26,28,29}$ Solicited reactogenicity was more frequently reported in both groups in cohort 1 , consistent with a reporting bias as this older age-group may have been better able to communicate feeling unwell. Abdominal pain and vomiting may have been more common in PXVX0200 recipients although in the vast majority of participants these were mild to moderate in severity. Diarrhea is a potentially important $A E$ with live oral cholera vaccine. However, in this study, it was reported with similar frequency by vaccine and placebo recipients, although severe diarrhea was noted in three $(0.9 \%)$ PVXV0200 recipients. These are similar to the rates of diarrhea seen in adults in the lot consistency study and in children aged 2-9 years with the previously commercialized form of CVD 103-HgR. ${ }^{24,28}$ There were no differences between vaccine and placebo recipients in the incidence of unsolicited AEs, and there were no vaccine-related SAEs.

Two other cholera vaccines are available outside of the United States, WC-rBS (Dukoral) and bivalent, killed wholecell vaccine (Shanchol, Euvichol), administered as two or three doses depending on age. ${ }^{50}$ As a single-dose immunization, PXVX0200 could offer a potential advantage in an outbreak setting in a vulnerable pediatric population.

\section{CONCLUSION}

These data demonstrate that PXVX0200 may be a safe, tolerable cholera vaccine option that produces a robust vibriocidal antibody response in children and adolescents aged 6-17 years and would be expected to provide protection against cholera in this pediatric population from developed countries who are at an increased risk of infection when traveling to or residing in at-risk countries.

Received March 29, 2019. Accepted for publication October 10, 2019.

Published online November 25, 2019.

Note: Supplemental table appears at www.ajtmh.org.

Acknowledgments: We are grateful to the participants and their families for volunteering, and investigators for recruitment and care of the participants: John Ervin, the Center for Pharmaceutical Research, Kansas City, MO; Terry Klein, Heartland Research Associates, Wichita, KS; Cynthia Strout, Coastal Carolina Research Center, Mt. Pleasant, SC; Matthew Davis, Rochester Clinical Research Inc, Rochester, NY; Carlos Fierro, Johnson County Clin-Trials, Lenexa, KS; Stephen Kebe, Aventiv Research, Ohio, Columbus, OH; Stephan Sharp, Clinical Research Associates Inc., Nashville, TN. Clinical Trials Registration: clinicaltrials.gov NCT 03220737.

Financial support: This research was funded entirely by Emergent Travel Health, a subsidiary of Emergent BioSolutions, Inc. This research did not receive any specific grant from funding agencies in the public or not-for-profit sectors.
Disclosure: All authors attest that they meet the AJTMH criteria for authorship.

Authors' addresses: James M. McCarty, Stanford University School of Medicine, Stanford, CA, E-mail: jmccart2@stanford.edu. Emma C. Gierman, Lisa Bedell, Michael D. Lock, and Sean Bennett, PaxVax, Inc., Redwood City, CA, E-mails: emmagierman@gmail.com, BedellL@ ebsi.com, mike@lockstats.com, and sbennett22903@gmail.com.

This is an open-access article distributed under the terms of the Creative Commons Attribution License, which permits unrestricted use, distribution, and reproduction in any medium, provided the original author and source are credited.

\section{REFERENCES}

1. Weil AA, Ryan ET, 2018. Cholera: recent updates. Curr Opin Infect Dis 31: 1-7.

2. Camacho A et al., 2018. Cholera epidemic in Yemen, 2016-18: an analysis of surveillance data. Lancet Glob Health 6: e680-e690.

3. Organization Pan American Health, 1991. Cholera situation in the Americas: an update. Epidemiol Bull 12: 1-14.

4. Newton A et al., 2011. Cholera in the United States associated with epidemic in Hispaniola. Emerg Infect Dis 17: 2166-2168.

5. Ali M, Nelson AR, Lopez AL, Sack DA, 2015. Updated global burden of cholera in endemic countries. PLoS Negl Trop Dis 9: e0003832.

6. Eberhart-Phillips $J$ et al., 1996. An outbreak of cholera from food served on an international aircraft. Epidemiol Infect 116: 9-13.

7. Enzensberger R, Besier S, Baumgartner N, Brade V, 2005. Mixed diarrhoeal infection caused by Vibrio cholerae and several other enteric pathogens in a 4-year-old child returning to Germany from Pakistan. Scand J Infect Dis 37: 73-75.

8. Reyes-Corcho A, Pinsker RW, Sarkar S, Bagheri F, Patel MC, Lam $P$, González A, 2012. Cholera gravis associated with acute renal failure in a traveler from Haiti to the United States. Travel Med Infect Dis 10: 236-239.

9. World Health Organization, 2017. Cholera vaccines: WHO position paper-August 2017. Wkly Epidemiol Rec 92: 477-500.

10. Mosley WH, Ahmad S, Benenson AS, Ahmed A, 1968. The relationship of vibriocidal antibody titre to susceptibility to cholera in family contacts of cholera patients. Bull World Health Organ 38: 777-785.

11. Mosley WH, McCormack WM, Ahmed A, Chowdhury AK, Barui RK, 1969. Report of the 1966-67 cholera vaccine field trial in rural East Pakistan. 2. Results of the serological surveys in the study population-the relationship of case rate to antibody titre and an estimate of the inapparent infection rate with Vibrio cholerae. Bull World Health Organ 40: 187-197.

12. Levine MM, Black RE, Clements ML, Cisneros L, Nalin DR, Young CR, 1981. Duration of infection-derived immunity to cholera. $J$ Infect Dis 143: 818-820.

13. Levine MM, Chen WH, Kaper JB, Lock M, Danzig L, Gurwith M, 2017. PaxVax CVD 103-HgR single-dose live oral cholera vaccine. Expert Rev Vaccines 16: 197-213.

14. Suharyono et al., 1992. Safety and immunogenicity of single-dose live oral cholera vaccine CVD 103-HgR in 5-9 year-old Indonesian children. Lancet 340: 689-694.

15. Simanjuntak $\mathrm{CH}$ et al., 1993. Safety, immunogenicity, and transmissibility of single-dose live oral cholera vaccine strain CVD 103-HgR in 24- to 59-month-old Indonesian children. J Infect Dis 168: 1160-1176.

16. Lagos R, Avendano A, Prado V, Horwitz I, Wasserman S, Losonsky G, 1995. Attenuated live cholera vaccine strain CVD 103-HgR elicits significantly higher serum vibriocidal antibody titers in persons of blood group O. Infect Immun 62: 707-709.

17. Lagos R, Losonsky G, Abrego P, San Martin O, Prado V, Wasserman S, Levine MM, 1996. Tolerance, immunogenicity, excretion and transmission of the live-attenuated cholera vaccine CVD 103-HgR. Double blind paired study in Chilean 24-to 59-month old children. Bol Med Hosp Infant Mex 53: 214-220.

18. Wiedermann G, Kollaritsch $H$, Jeschko E, Kundi M, Herzog C, Wegmuller B, 1998. Adverse events after oral vaccination against cholera with CVD 103-HgR. Wien Klin Wochenschr 110: 376-378. 
19. Lagos R, Fasano A, Wasserman SS, Prado V, San Martin O, Abrego P, Losonsky GA, Alegria S, Levine MM, 1999. Effect of small bowel bacterial overgrowth on the immunogenicity of single-dose live oral cholera vaccine CVD 103-HgR. J Infect Dis 180: $1709-1712$.

20. Lagos R, Martin OS, Wasserman SS, Prado V, Losonsky GA, Bustamante C, Levine MM, 1999. Palatability, reactogenicity and immunogenicity of engineered live oral cholera vaccine CVD 103-HgR in Chilean infants and toddlers. Pediatr Infect Dis J 18: 624-630.

21. Richie E, Punjabi NH, Sidharta Y, Peetosutan K, Sukandar M, Wasserman SS, 2000. Efficay trial of single-dose live oral cholera vaccine CVD 103-HgR in North Jakarta, Indonesia, a cholera endemic area. Vaccine 18: 2399-2410.

22. Cooper PJ, Chico ME, Losonsky G, Sandoval C, Espinel I, Sridhara R, 2000. Albendazole treatment of children with ascariasis enhances the vibriocidal antibody response to the live attenuated oral cholera vaccine CVD 103-HgR. J Infect Dis 182: 1199-1206.

23. Faucher JF, Binder R, Missinou MA, Matsiegui PB, Grus $H$, Neubauer R, 2002. Efficacy of atovaquone/proguanil for malaria prophylaxis in children and its effect on the immunogenicity of live oral typhoid and cholera vaccines. Clin Infect Dis 35: 1147-1154.

24. Swiss Serum and Vaccine Institute Berne. Orochol [package insert]; 2000.

25. Chen WH, Greenberg RN, Pasetti MF, Livio S, Lock M, Gurwith M, Levine MM, 2014. Safety and immunogenicity of single-dose live oral cholera vaccine strain CVD 103-HgR, prepared from new master and working cell banks. Clin Vaccine Immunol 21: 66-73.

26. Chen WH et al., 2016. Single-dose live oral cholera vaccine CVD 103-HgR protects against human experimental infection with Vibrio cholerae O1 El Tor. Clin Infect Dis 62: 1329-1335.

27. Haney DJ, Lock MD, Simon JK, Harris J, Gurwith M, 2017. Antibody-based correlates of protection against cholera analysis of a challenge study in a cholera-naive population. Clin Vaccine Immunol 24: 1-11.

28. McCarty JM, Lock MD, Hunt KM, Simon JK, Gurwith M, 2018. Safety and immunogenicity of single-dose live oral cholera vaccine strain CVD 103-HgR in healthy adults age 18-45. Vaccine 36: 833-840.

29. McCarty JM, Lock MD, Bennett S, Hunt KM, Simon JK, Gurwith $M, 2019$. Age related immunogenicity and reactogenicity of live oral cholera vaccine CVD 103-HgR in a randomized, controlled clinical trial. Vaccine 37: 1389-1397.

30. Sack DA, Qadri F, Svennerholm AM, 2008. Determinants of responses to oral vaccines in developing countries. Ann Nestle [Engl] 66: 71-79.

31. Levine MM, 2010. Immunogenicity and efficacy of oral vaccines in developing countries: lessons from a live cholera vaccine. $B M C$ Biol 8: 129 .

32. Czerkinsky C, Holmgren J, 2015. Vaccines against enteric infections for the developing world. Philos Trans $R$ Soc Lond B Biol Sci 370: 20150142.

33. Lim J, 2011. Hedonic scaling: a review of methods and theory. Food Qual Prefer 22: 733-747.

34. Beneson AS, Saad A, Mosley WH, 1968. Serological studies in cholera. 2. The vibriocidal antibody response of cholera patients determined by a microtechnique. Bull World Health Organ 38: 277-285.

35. Agresti A, Coull BA, 1998. Approximate is better than "exact" for interval estimation of binomial proportions. Am Stat 52: 119-126.

36. Mascarello M, Deiana M, Maurel C, Lucarelli C, Luzzi I, Luzzait R, 2013. Cholera with severe renal failure in an Italian tourist returning from Cuba, July 2013. Euro Surveill 18: 20572.

37. Slesak G, Fleck R, Jacob D, Grunow R, Schafer J, 2016. Imported cholera with acute renal failure after a short business-trip to the Philippines, Germany, October 2015. Euro Surveillance 21:1-3.

38. Fritzell B, 1998. Bridging studies. Dev Biol Stand 95: 181-188.

39. Mosley WH, Beneson AS, Barui R, 1968. A serological survey for cholera antibodies in rural East Pakistan. 2. A comparison of antibody titres in the immunized and control population of a cholera-vaccine field-trial area and the relation of antibody titre to cholera case rate. Bull World Health Organ 38: 335-346.

40. Glass RI, Svennerholm A, Khan MR, Huda S, Huq MI, Holmgren J, 1985. Seroepidemiologic studies of El Tor cholera in Bangladesh: association of serum antibody levels with protection. J Infect Dis 151: 236-242.

41. Levine MM, Kaper JB, 1995. Live oral cholera vaccine: from principle to product. Bull Inst Pasteur 93: 243-253.

42. Tacket CO, Losonsky G, Nataro JP, Cryz SJ, Edelman R, Kaper JB, Levine MM, 1992. Onset and duration of protective immunity in challenged volunteers after vaccination with live oral cholera vaccine CVD 103-HgR. J Infect Dis 166: 837-841.

43. Tacket CO, 1999. Randomized, double-blind, placebo-controlled, multicentered trial of the efficacy of a single dose of live oral cholera vaccine CVD 103-hgr in preventing cholera following challenge with Vibrio cholerae O1 El Tor Inaba three months after vaccination. Infect Immun 67: 6341-6345.

44. Levine MM, Kaper JB, Herrington D, Ketley J, Losonsky G, Tacket $C, 1988$. Safety, immunogenicity and efficacy of recombinant live oral cholera vaccines CVD 103 and CVD 103-HgR. Lancet 2: 467-470.

45. Cryz S, Levine MM, Kaper JB, Furer E, Althaus B, 1990. Randomized, double-blind, placebo-controlled trial to evaluate the efficacy and immunogenicity of the live oral cholera vaccine CVD 103-HgR in Swiss adults. Vaccine 8: 577-580.

46. Kotloff W, Wasserman SS, O'Donnell S, Losonsky GA, Cryz SJ, Levine MM, 1992. Safety and immunogenicity in North America of a single dose of live oral cholera vaccine CVD 103-HgR: results of a randomized, placebo-controlled, double-blind cross-over trial. Infect Immun 60: 4430-4432.

47. Migasena S, Pitisuttitham $P$, Prayurahong $P$, Suntharasami $P$, Supanaranond W, Desakorn V, 1989. Preliminary assessment of the safety and immunogenicity of live oral cholera vaccine strain CVD 103-HgR in health Thai adults. Infect Immun 57: 3261-3264.

48. Temik R, Liu F, Bartlett JA, Khong YM, Tan DCT, Dixit T, Wang S, Galella EA, Goa Z, Klein S, 2018. Assessment of swallowability and palatability of oral dosage forms in children: report from an M-CERSI pediatric formulation workshop. Int J Pharm 536: 570-581.

49. Mistry P, Batchelor H; SPaeDD-UK project, 2017. Methodology used to assess acceptability of oral pediatric medicines: a systematic literature search and narrative review. Pediatr Drugs 19: 223-233.

50. Saha A, Rosewell A, Hayen A, Maclntyre CR, Qadri F, 2017. Improving immunization approaches to cholera. Expert Rev Vaccines 16: 235-248. 